\title{
Sputtered Indium Tin Oxide thin films deposited on glass substrate for photovoltaic application
}

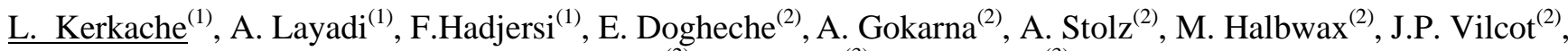 \\ D.Decoster ${ }^{(2)}$ B. El Zein ${ }^{(3)}$, S. S. Habib ${ }^{(3)}$ \\ (1) Département de Physique, Faculté des Sciences, Université Ferhat Abbas Sétif 19000, Algérie. \\ Phone/Fax number:+00 213369379 43, e-mail: kerkachelaid@yahoo.fr \\ (2) Institut d'Electronique Microélectronique et Nanotechnologie, IEMN-CNRS UMR 8520 Université de Valenciennes et \\ du Hainaut Cambrésis, Cité scientifique F- 59000 Lille, France. \\ (3) Center of Nanotechnology, King Abdul Aziz University, P.O. Box 80204, Jeddah 21589, Saudi Arabia
}

\begin{abstract}
Polycrystalline indium tin oxide (ITO) films were prepared on glass substrates by radio frequency (RF) sputtering system from a high density target $\left(90 \mathrm{wt} . \mathrm{In}_{2} \mathrm{O}_{3}\right.$ and 10 wt. $\% \mathrm{SnO}_{2}$ ). X-ray diffraction (XRD) and Scanning Electron Microscopy (SEM) experiments were performed to study the structure and the surface morphology of these samples. The results show that the as-deposited ITO thin films deposited on glass have a $\langle 100\rangle$ texture, as the film grows the preferred orientation changes from $\langle 100\rangle$ to $\langle 111\rangle$. The lattice parameters are found to be larger than the bulk value, indicating that the samples are under a tensile stress. The grain size increases with increasing thickness. SEM images show a dense granular structure with grains having different shapes and sizes. After deposition, the samples have been annealed in air at temperature $\mathrm{T}$ around $400^{\circ} \mathrm{C}$ for 1 hour. We found that the $<111\rangle$ texture becomes stronger after the annealing treatment. A large increase in the grain size after annealing is also observed. The lattice constant decreases with $\mathrm{T}$ to become closer to the bulk value, i.e. annealing seems to relieve the stress present in the as-deposited films. The optical transmisión is greater than $80 \%$ in the visible region. The refractive index $n$ values are found to be in the 1.7-1.9 range. The energy gap values are between 3.58 and $3.88 \mathrm{eV}$.
\end{abstract}

\section{Key words}

ITO film, RF sputtering, heat treatment, structural and optical properties.

\section{Introduction}

Tin-doped indium oxide $\mathrm{In}_{2} \mathrm{O}_{3}$ : $\mathrm{Sn}$ (ITO) is a highly degenerate n-type wide gap semi-conductor (band gap $3.7 \mathrm{eV}$ ) and belongs to the class of transparent conductive oxides (TCO) which is important for photovoltaic applications. ITO film has a low electrical resestivity, transparency in the visible and high infrared reflectivity.
Because of these characteristics, ITO films have been widely used in solar cells [1], conductive electrodes [2] and ohmic contacts light emitting diodes [3].

There are many deposition techniques to obtain high quality ITO films such as RF and DC sputtering [4-5] sol-gel [6] and pulsed laser deposition [7].

In the present work, ITO films were deposited by RF sputtering. We have set the power at $250 \mathrm{~W}$ and changed the thickness from $171 \mathrm{~nm}$ to $513 \mathrm{~nm}$. Also the effect of annealing at $\mathrm{T}=400^{\circ} \mathrm{C}$ is investigated, these annealing temperature $\left(400^{\circ} \mathrm{C}\right)$ has been chosen, since it was found in a previous work [8] that with this temperature, we obtain a practically stress free samples. The morphology, structural and optical properties of ITO films were examined.

\section{Experimental methods}

The ITO thin films have been deposited by RF sputtering onto glass substrate from a ceramic $\mathrm{In}_{2} \mathrm{O}_{3}: \mathrm{SnO}_{2}$ target (90:10Wt. \%, 99,99 \%). The pressure, before deposition, is about $10^{-7}$ mbar. The highest power value $\mathrm{P}$, delivered by the generator is $3000 \mathrm{~W}$ at radio frequency (13.56 $\mathrm{MHz}$ ). The samples have been prepared at power values $\mathrm{P}=250 \mathrm{~W}$ and for different thickness (171 to $513 \mathrm{~nm}$ ). Structural properties have been studied by means of the $\mathrm{X}$-ray diffraction technique and the Scanning Electron Microscopy (SEM). A SIEMENS DR 5000 type X-ray diffractometer working in the $2 \theta$ mode and with the $\mathrm{K} \alpha$ $\mathrm{Cu}$ radiation have been used. The transmission curves were obtained by the means of Perkin-Elmer type spectrometer; these experiments allowed us to get the refractive indices (n), the thickness and the energy gap (Eg) of these films. The annealing experiments were done, in the air, at temperature $\mathrm{T}=400^{\circ} \mathrm{C}$ for 1 hour. 


\section{Results and discussions}

\subsection{Structural properties}

$\mathrm{X}$-ray diffraction spectra have been used to determine the texture, the grain size and the lattice parameters. Examples of X-ray spectra are shown in Fig.1.

The lattice parameter (a) has been deduced from the peak position, using the strongest peak, i.e. the (222) in the spectrum. Note that the powder lattice constant of $\operatorname{In}_{2} \mathrm{O}_{3}$ is equal to $10.118 \AA$ [9]. The lattice parameters in these samples are somewhat higher than the powder value, indicating that the samples are strained; this strain is probably due to a growth induced stress. In Fig.2, we show the variation of the lattice parameter (a) for as deposited and annealed (at $\mathrm{T}=400{ }^{\circ} \mathrm{C}$ ) samples. The lattice constant values become closer to the bulk value. This after annealing indicates that the stress, present in the as- deposited films, have been relieved after annealing.

The grain sizes were derived from the X-ray diffraction spectra following the Scherrer method [10]. The grain size $\mathrm{D}$ is given by:

$$
\mathrm{D}=\frac{K \lambda}{\Delta(2 \theta) \cos (\theta)}
$$

Where $\lambda$ is the $\mathrm{X}$-ray wavelength $(\lambda=1.5406 \AA)$, the constant $\mathrm{K}=180 / \pi, \theta$ is the diffraction angle, the angle at which the peak of a particular orientation occurs

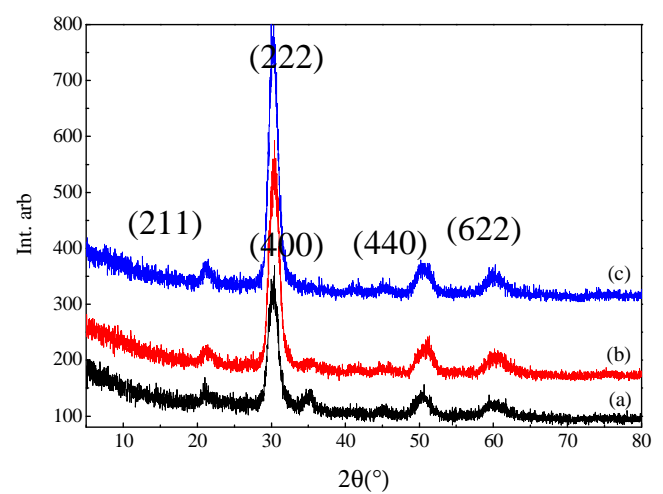

Fig.1: X-ray spectra of ITO thin films with thickness equal (a) $171 \mathrm{~nm}$, (b) $333 \mathrm{~nm}, \quad$ (c) $513 \mathrm{~nm}$.

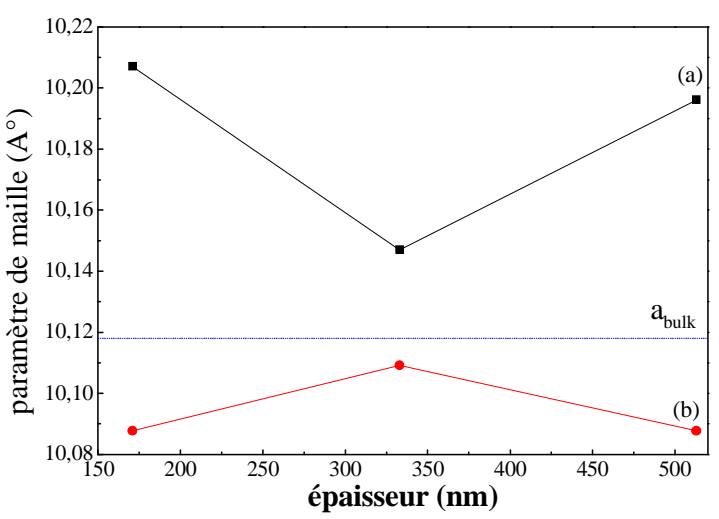

Fig.2 Lattice parameter vs thickness for a) $\mathrm{RT}$ (b) $\mathrm{T}=400^{\circ} \mathrm{C}$
Grain sizes and shapes have also been observed by means of the SEM. In fig.3, we show an SEM image for the sample made at $\mathrm{P}=250 \mathrm{~W}(\mathrm{t}=171 \mathrm{~nm})$. We can observe a dense granular structure. The grains have different shapes and sizes. The grain size found using SEM is larger than those derived from X-ray diffraction.

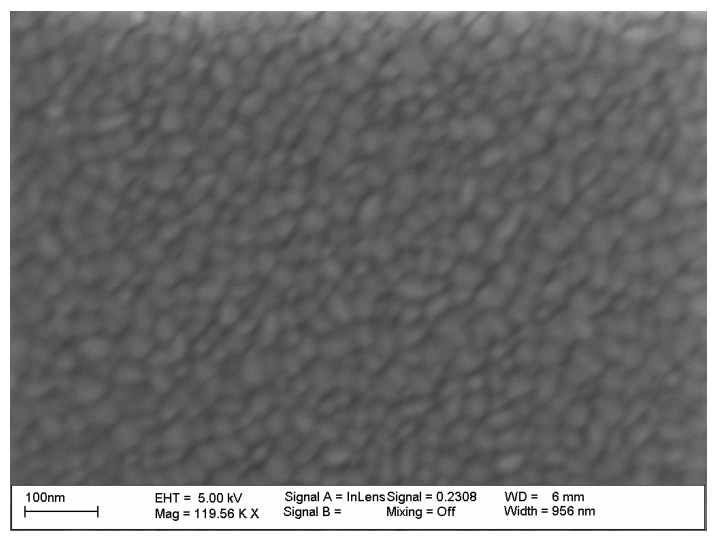

Fig..3 Typical SEM image of ITO film with $\mathrm{t}=171 \mathrm{~nm}$ deposited on $\mathrm{Si}$

\subsection{Optical properties}

Optical transmission (T) spectra measurements taken at normal incidence were performed in the UV-Visible range to analyze the optical properties of the ITO films on glass substrates. High transparency of ITO thin films in the visible region had quite an effect on their applications in the photovoltaic devices. Owing to this nature, transmittance is considered as one of the essential properties in evaluating the optical performance of ITO films. In the present study, it was found that the transmission expanded after annealing at $\mathrm{T}=400^{\circ} \mathrm{C}$ for 1 hour.Fig.4 shows the optical transmission for ITO film with a thickness of $513 \mathrm{~nm}$ as deposited and annealing at $\mathrm{T}=400^{\circ} \mathrm{C}$. The average optical transmission in the visible light region improves with heat treatment, exhibiting $80 \%$ for film at RT and $85 \%$ after annealing at $\mathrm{T}=400^{\circ} \mathrm{C}$. The improvement of optical properties caused by increasing heat treatment temperature is due to the enhanced formation and crystallization of the ITO films. Higher temperature heat treatment leads to a better crystallization and lower level of defects near the grain foundries, thus resulting in the improvement of structural homogeneity and the decrease of light reflection [11].

The energy gap was obtained from the transmission curves. In the strong absorption region, the UV region, the direct energy gap Eg is related to the absorption coefficient $\alpha$ by:

$$
\alpha=\mathrm{A}(\mathrm{h} v-\mathrm{Eg})^{1 / 2}
$$

A being a constant, $v$ is the frequency; by plotting $\alpha^{2}$ vs. $\mathrm{h} v$ in the region where (2) is valid, i.e. in the region of strong absorption, the UV region, we get $\mathrm{E}_{\mathrm{g}}$ by extrapolation of the straight line to $\alpha=0$. In Fig. 5, we plot $\alpha^{2}$ vs. the incident energy hv for all samples. We note that the curves are straight in the UV region. We note that 
the values of the energy gap are between $3.58 \mathrm{eV}$ and $3.88 \mathrm{eV}$.

The absorption coefficient $\alpha$ was determined from the relation $I=I_{0} \exp (-\alpha \mathrm{t})$ where $t$ is the thickness of the sample, I the transmitted intensity and $\mathrm{I}_{0}$ the incident intensity. This relation gives $\alpha=(2.303 / \mathrm{t}) \log _{10}$ $\left(\mathrm{I}_{0} / \mathrm{I}\right)$.

From the transmission spectra, we measure ratio of transmission $\mathrm{T}\left(\mathrm{T}=\mathrm{I} / \mathrm{I}_{0}\right)$. The transmission curves present a series of maximum (noted $\mathrm{T}_{\mathrm{M}}$ ) and minimum $\left(\mathrm{T}_{\mathrm{m}}\right)$ of the envelope of interference, are now considered to be continuous functions of $\lambda$. For any $\lambda, T_{M}$ has a correspondent value $\mathrm{T}_{\mathrm{m}}$. The refractive index is calculated by:

$$
\mathrm{n}=\left[\mathrm{N}+\left(\mathrm{N}^{2}-\mathrm{n}_{\mathrm{s}}^{2}\right)^{1 / 2}\right]^{1 / 2}
$$

where $\mathrm{N}$ is defined as

$\mathrm{N}=\left(\mathrm{n}_{\mathrm{s}}^{2}+1\right) / 2+2 \mathrm{n}_{\mathrm{s}}\left(\mathrm{T}_{\mathrm{M}}-\mathrm{T}_{\mathrm{m}}\right) / \mathrm{T}_{\mathrm{M}} \mathrm{T}_{\mathrm{m}}$.

Following this method, we have derived values for the refractive index $\mathrm{n}$ which have been found to be in the range 1.7-1.9.

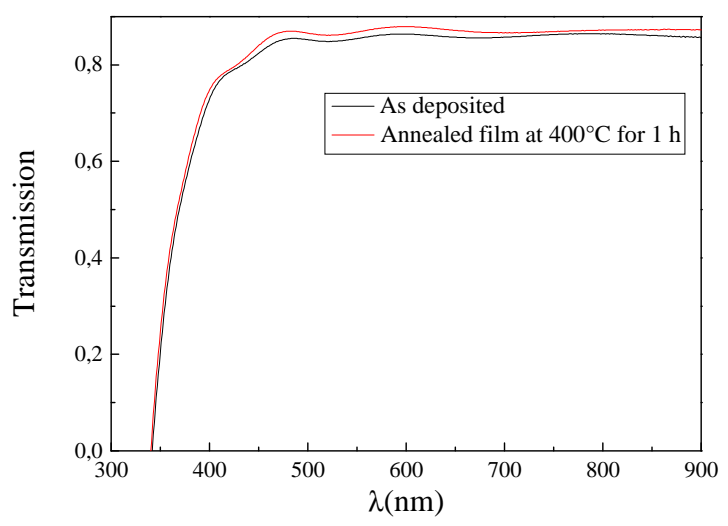

Fig.4 Transmission curves for samples at RT and annealing at $\mathrm{T}=400^{\circ} \mathrm{C}$

\section{Conclusion}

Structural and optical properties of RF sputtered ITO thin films and the effects of annealing have been studied. From X-ray diffraction, we observed that thinner films have a $\langle 100\rangle$ texture, as the growth, the preferred orientation is $\langle 111\rangle$ and the $\langle 111\rangle$ texture becomes stronger as the thickness increases. The lattice parameters are found to be larger than corresponding to the power indicating that the samples are under a tensile stress. SEM images show a dense granular structure with grains having different shapes and sizes. The energy gap Eg, computed from the transmission curve, was found to decrease from 3.58 to $3.88 \mathrm{eV}$ as the thickness increases. Annealing experiments were done, in the air, at temperature $\mathrm{T}=400{ }^{\circ} \mathrm{C}$ for 1 hour, we found that the $<111>$ texture become stronger after annealing i.e. the annealing favours the $\langle 111\rangle$ texture of the grain size with increasing $\mathrm{T}$ is observed. where $n_{s}$ is the refractive index of glass substrate (in our case $n_{s}=1.5$ ).

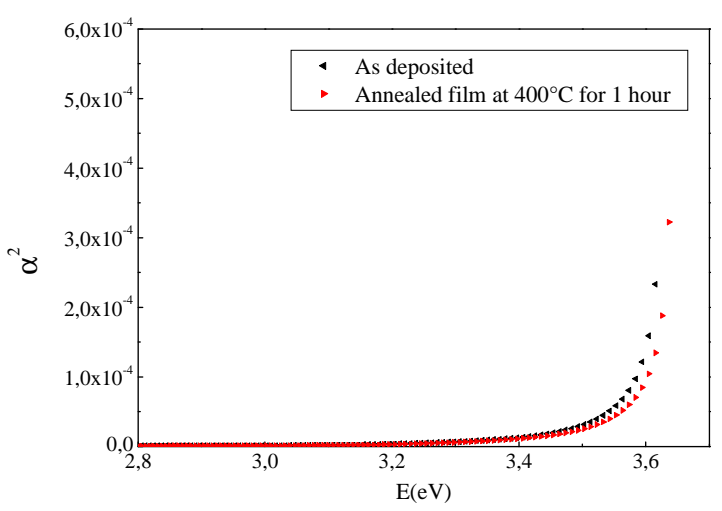

Fig.5 Optical band gap of ITO films

\section{References}

[1] ] B. Zimmermann, M. Glatthaar, M. Niggermann, M.K. Riede, A. Hinsch, A. Gombert, Solar Energy Materials \&Solar Cells 91 (2007)374.

[2] V. Bornand, Ph. Papet, Mater. Chem. Phys. 92 (2005) 424.

[3] H.H Yu, S-J Hwang, M-C Teng, C-C Tseng. Optics Communications 259(2006)187-193.

[4] M. Nisha, M.K. Jayaraj, Appl.Surf.Sci 255(2008)1790-1795

[5] L. Kerkache, A. Layadi, A. Mosser, journal of Alloys and Compounds 485(2009) 46-50

[6] T.F. Stoica, V.S. Teodorescu, M.G. Blanchin, M. Gortner, M. Losurdo, M. Zaharescu, Mater. Sci. Eng. B101 (2003)222.

[7] J. B. Choi, J. H. Kim, K.A. Jeon, S. Y. Lee, Mat.science and Eng. B102 (2003) 376-379.

[8] L. Kerkache, A. Layadi, E. Dogheche, D. Remiens, J.Phys.D:Appl.Phys.39, 184 (2006)

[9] R.N Jochi, V.P Singh, J.C McClure, Thin solid films 257 (1995) 32-35.

[10] A. El Hichou, A. Kachouane, J.L Budendorff, M. Addou, J. Ebothe, M. Troyon, A. Bougrine, Thin Solid Films 458, 263 (2004)

[11] Z.H Li, Y.P Ke, D.Y Ren, Trans Nonferrous Met. Soc. China 18(2008)366-371. 